\title{
Influence of External Jet on Hydraulic Performance and Flow Field Characteristics of Water Jet Propulsion Pump Device
}

\author{
Jinxin Wu, Li Cheng ${ }^{(D}$, Can Luo, and Chuan Wang $(i D)$ \\ College of Hydraulic Science and Engineering, Yangzhou University, Yangzhou 225009, China \\ Correspondence should be addressed to Li Cheng; chengli@yzu.edu.cn and Chuan Wang; wangchuan198710@126.com
}

Received 16 December 2020; Revised 6 March 2021; Accepted 11 May 2021; Published 24 May 2021

Academic Editor: Peter Mucka

Copyright ( $\odot 2021$ Jinxin Wu et al. This is an open access article distributed under the Creative Commons Attribution License, which permits unrestricted use, distribution, and reproduction in any medium, provided the original work is properly cited.

\begin{abstract}
Water jet propulsion technology has broad application prospects in the field of ships, and water jet technology is a kind of high and new technology that is booming and has a wide range of applications. However, there are a few studies on the effect of the external jet on the performance of the water jet propulsion pump, and it is urgent to carry out this research. In this paper, the standard $k-\varepsilon$ turbulence model is used to carry out the numerical simulation study of the influence of the external jet on the hydraulic performance and flow field characteristics of the water jet propulsion pump device. This paper discusses the selection of calculation models, the division of grids and the setting of turbulence models, and an in-depth analysis of the calculation results. The research results show that when a high-speed water jet enters a moving water body, it will cause turbulence in the moving water body. With the increase of jet flow, the turbulence phenomenon will be improved. The average velocity of the outlet section of the nozzle is consistent with the change of the total pressure. The average vortex gradually decreases, the turbulent kinetic energy changes little, the turbulence dissipation first decreases and then increases, and the nozzle axial force changes more and more. The axial force and thrust of the device will obviously increase when the two water streams merge and spray, and they will increase with the increase of the jet flow rate. By revealing the influence mechanism of the external jet on the water jet propulsion pump device, it can provide a theoretical basis and guiding direction for further optimizing the hydraulic performance of the entire device.
\end{abstract}

\section{Introduction}

Due to the superiority of water jet propulsion technology, more high-speed ferries, commercial ships, and military vessels use water jet propulsion pump devices. Scholars at home and abroad have also conducted a large number of experimental studies and numerical simulation studies on the water jet propulsion pump device, which has made the water jet propulsion technology a breakthrough development in recent decades [1-3]. With the rapid development of CFD technology, numerical simulation has become an important research method and has been widely used in different research directions [4-7].

Water jet technology is a rapidly emerging new technology, and it has a broad prospect in the application of water jet thrusters. Zhang et al. [8] reviewed the two main applications of the current underwater gas-liquid two-phase propulsion technology-gas-liquid two-phase ramjet and bubble afterburner water jet propulsion engine theory and experimental research, and Point out its main development trend. Meng et al. [9] used fluid dynamics (CFD) technology to study the flow field structure evolution and mixing efficiency in jet mixing equipment, analyzed the parameters that affect the jet mixing efficiency, and summarized the mixing time correlations proposed by different researchers. Yu et al. [10] established two sets of models by means of comparative analysis and studied the thrust of water jet thrusters with and without pressure chambers using simulation analysis methods and experimental verification methods. The size of the pressure chamber has been drawn to the conclusion of the influence of the thrust of the water jet thruster. Wu et al. [11] carried out detailed experimental measurements on the three-dimensional flow structure and turbulence in the tip area of the water jet axial flow pump and combined the RANS method and the LES method to carry out deep CFD numerical calculations. Jiao et al. [12] conducted a hydraulic optimization design and performance study on the flow channel of the water jet propulsion pump. 
Kinnas and Lee [13] used the internally developed ship CFD program CFD SHIP-IOWA to conduct an in-depth study on the hydrodynamics and propulsion performance of the water jet propulsion ship system and used the overlapping grid technology to realize the longitudinal movement of the ship during navigation. Wang et al. [14, 15] proposed an optimized design method for pumps. He et al. [16] and Zhang et al. [17] studied the numerical calculation method of the pump and gave the corresponding experimental results. Wang et al. [18-20] studied the factors affecting pump performance through numerical calculations. Zhou et al. $[21,22]$ used the computational fluid dynamics method to analyze the water flow characteristics of the pump. Duan et al. [23] conducted numerical simulation research and prediction on the cavitation characteristics of water jet propulsion pumps. Jin [24] conducted three-dimensional design and numerical experiments on water jet propulsion pumps. Wang et al. [25] conducted a numerical simulation study on impinging water jets by selecting a turbulence model. Chang and Wang [26] used the K-flow turbulence model to predict the propulsion performance of water jets. Xia et al. [27] studied the hydraulic characteristics of the rotating stall of the water jet propulsion system and proposed corresponding suppression measures.

At present, there are a few studies on a high-speed water jet entering a moving water body at home and abroad, and the understanding of the effect of the external jet on the performance of the pump is not very clear. Therefore, it is necessary to conduct in-depth research on the performance of the water jet propulsion pump device with an external jet. This paper is based on the standard $k-\varepsilon$ turbulence model to numerically simulate the flow field of a water jet propulsion pump device with an external jet and use the velocity, total pressure, vorticity, turbulent kinetic energy cloud image, and other flow field information data of the nozzle outlet section and the longitudinal section of the nozzle section. By intuitive observation of the internal flow characteristics of the nozzle section of the propulsion pump, this paper reveals the influence mechanism of the external jet and provides a theoretical basis and guiding direction for further optimizing the hydraulic performance of the entire device.

\section{Water Jet Propulsion Pump Model and Numerical Simulation Method}

2.1. Geometric Model and Computational Domain. This paper selects a certain type of water jet propulsion pump device as the research object. The water jet propulsion pump device is mainly composed of a water inlet channel, propulsion pump, nozzle, and reversing device. The water inlet channel is one of the main components of the water jet propulsion device, and its function is to transfer the water from the bottom of the ship to the propulsion pump. The propulsion pump is the core component of the water jet propulsion system. The mixed-flow pump type used in this article is composed of four parts: the impeller ( 6 pieces), the guide vane body (7 pieces), the inlet section, and the outlet section. It is mainly divided into two parts: the inlet passage and the propulsion pump. In order to study the effect of the external jet on the performance of the water jet propulsion pump device, a slight modification is made on the original selected model. A jet pipe is introduced from the small auxiliary pump through any guide vane (multiple guide vanes can be selected as required) into the impeller body and ejected jet through the water guide cone. The jet flow and the original water flow merge and eject, and the schematic diagram of the three-dimensional model of the water jet propulsion pump device with the jet flow is shown in Figure 1. (1) Figure 1 is only a schematic diagram of the model of the additional jet device. The specific auxiliary pump layout can be adjusted according to the actual situation. The focus of this article is not to study the additional jet device, but the research based on a device innovation. Study the phenomenon of a high-speed water jet entering a moving water body. (2) The idea of this additional jet device is to solve the problem that the ship needs to increase the instantaneous thrust when the hull space is limited. Therefore, when the space size is limited and two units cannot be arranged, considering the use of this additional jet device can increase the thrust and increase the sailing speed.

Affected by the hull boundary layer, incoming flow velocity, and pressure, the water flow entering the inlet passage is uneven. Therefore, when selecting the calculation fluid area, the fluid in the area around the inlet of the inlet passage at the bottom of the ship should also be included. Therefore, the calculation area includes three parts: the propulsion pump, the inlet passage, and the hull area, as shown in Figure 2(a). Take a circular cross section at the water guide cone of the impeller and define it as the outlet of the additional jet, as shown in Figures 2(b) and 2(c).

2.2. Mesh Generation. The quality of the grid is very important to the numerical simulation results of the water jet propulsion pump device. In this paper, ICEM CFD software is used to divide the entire calculation domain into a structured grid. The water inlet channel, the bottom area of the ship, the impeller, the guide vane, and the nozzle are all hexahedral structured grids, as shown in Figure 3. After the above grid is divided into blocks, each block is output as a CFX file, and each part is merged in the CFX preprocessing, and the boundary area and solution conditions are set and finally calculated.

2.3. Turbulence Model and Boundary Conditions. In this paper, the standard $k-\varepsilon$ turbulence model is used to solve the complex flow field in the water jet propulsion pump device with an external jet. Among them, the inlet of the device model is set as the speed inlet, which is initially set to $10 \mathrm{~m} / \mathrm{s}$; the outlet is the average static pressure outlet; set the reference pressure to $1 \mathrm{~atm}$; set the interface to transfer the values between the components; set the solid wall to the nonslip boundary condition, and use the standard function to process. The speed is initially set to $700 \mathrm{r} / \mathrm{min}$, and the calculated residual control value is set to $10^{-5}$.

2.4. Grid Independence Analysis of the Impeller Domain. In theory, the more the number of grids in the model, the smaller the solution error caused by the grids [28]. However, 


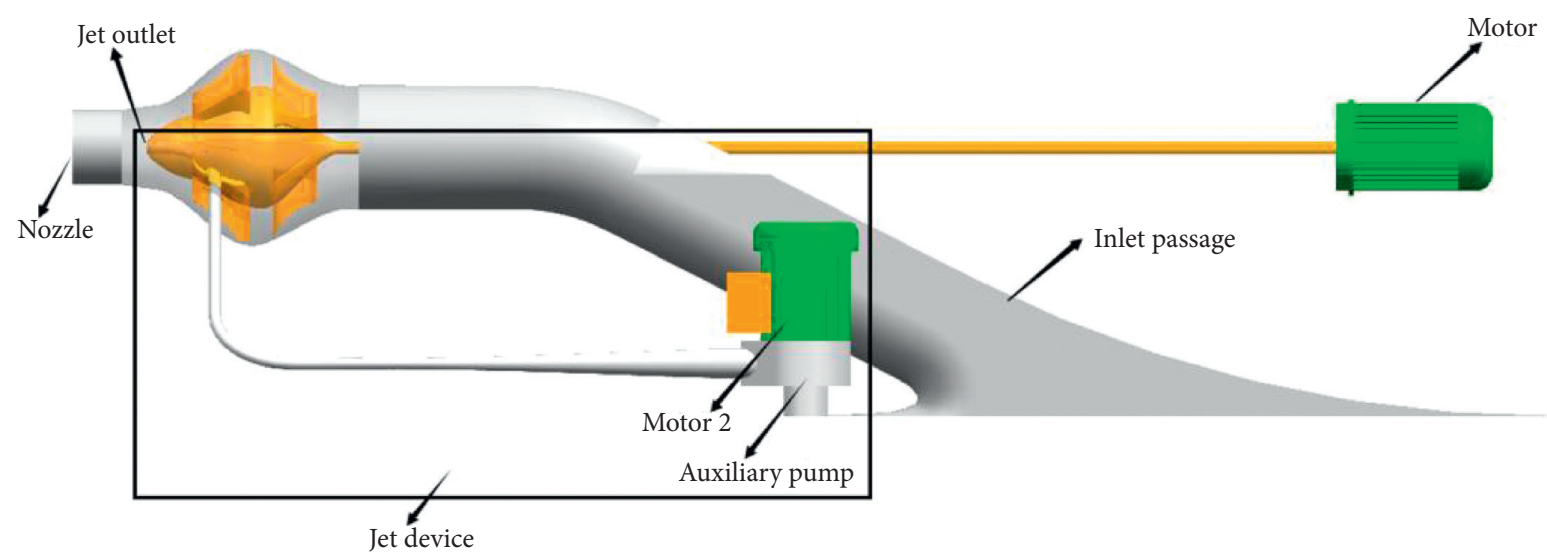

FIGURE 1: Schematic diagram of the geometric model of the water jet propulsion pump device with additional jet.

as the number of grids increases, the requirements for computer configuration also increase, and the calculation speed will also slow down. Therefore, on the basis of ensuring the accuracy of the solution in a certain range, the number of grids cannot be too large. For this reason, 6 sets of grids are selected for grid independence analysis, and the 6 sets of grids selected are shown in Table 1.

Take the head coefficient $H$ and efficiency $\eta$ of the water jet propulsion pump section as the main evaluation indexes. With the gradual increase in the number of grids, the changes in the head and efficiency of the propulsion pump section obtained by the numerical simulation gradually decrease, especially the relative error between the fifth set of grids and the sixth set of grids hardly changes, as shown in Figure 4 .

2.5. Research Plan. Assuming that the circular cross-sectional area of the jet outlet at the water guide cone is $30.77 \%$ of the cross-sectional area of the nozzle outlet (i.e., for the water jet propulsion pump device model selected in this article, the diameter of the circular cross-sectional jet outlet is $40 \mathrm{~mm}$ ), which can be defined by the velocity of the jet outlet changing the flow rate of the external jet, and the effects of different external jet flow rates on the hydraulic performance and flow field characteristics of the water jet propulsion pump device at different speeds are studied, as shown in Table 2.

\section{Analysis and Discussion of Calculation Results}

3.1. Hydraulic Performance Analysis. Because the numerical simulation results may not be able to simulate the actual situation well, it is necessary to verify the reliability of the numerical simulation results through experimental verification. However, it is difficult to test and verify this new type of additional jet device. Therefore, this paper selects the model of the water jet propulsion pump device before the jet is added to conduct experimental research to verify the reliability of the numerical simulation of this model, and indirectly verify the reliability of this model. Indirectly verify the reliability of the numerical simulation of the additional jet model with a slight modification on this model. Select different flow conditions for calculation, and get the flow and efficiency under the optimal conditions. In order to compare the calculation results under various working conditions more conveniently and intuitively, the flow, efficiency, and head of the optimal efficiency point are used as the benchmark, and the flow, efficiency, and head of other flow points are normalized by dimensions. According to the experimental data in the literature, combined with the numerical simulation result data in this paper, Figure 5 is obtained. It can be seen from Figures 5(a) and 5(b) that the numerical simulation results are in good agreement with the experimental results, so the simplified model above can be used for further analysis and research. In the figure, the best working condition BEP (best efficiency point) of the propulsion pump refers to the working condition corresponding to the highest efficiency point. $Q$ represents the flow rate, $Q_{\mathrm{BEP}}$ represents the flow rate corresponding to the highest efficiency point, $\eta$ represents the efficiency, and $\eta_{\mathrm{BEP}}$ represents the efficiency corresponding to the highest efficiency point, $H$ represents the head, and $H_{\mathrm{BEP}}$ represents the head corresponding to the highest efficiency point.

Figure 6 shows the relationship between the head of the propulsion pump section and the flow rate of the external jet at different speeds. Observing Figure 6, we can find that, as the jet flow increases, the head of the propulsion pump section gradually increases, and the rate of change of the head becomes larger. Under the same jet flow, the head of the propulsion pump section increases with the increase in speed. In the figure, $Q_{j} / Q_{O}$ represents the ratio of the flow at the coordinate point to the flow at the $z$ coordinate of 0 , and $H_{j} / H_{O}$ represents the ratio of the head at the coordinate point to the head at the $z$ coordinate of $0 . V_{S}$ means ship speed.

Figure 7 shows the velocity change curve on the central axis of the nozzle section at different jet velocities. Observing the curve, we can see that, with the increase of jet flow rate, the relative velocity change range from the center of the jet exit section to the center of the nozzle exit section also increases. The relative velocity at the same point increases with the increase of jet flow. In the figure, $z$ represents the $z$ 


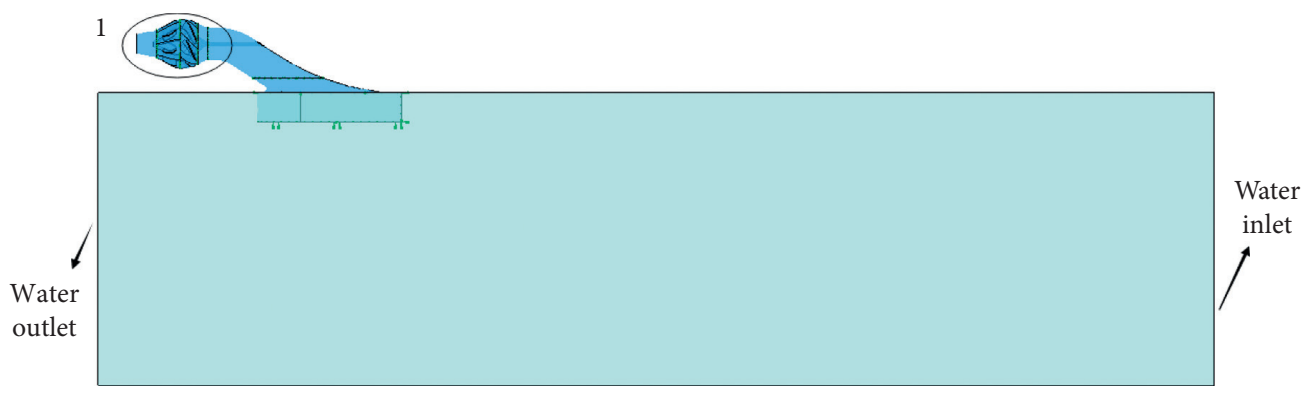

(a)

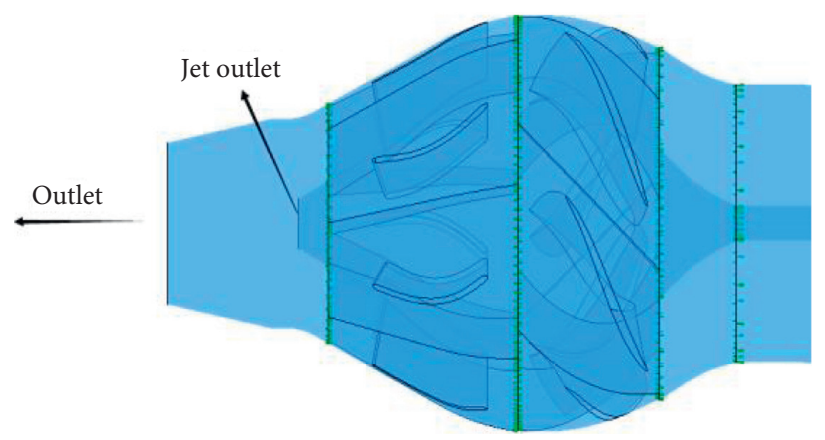

(b)

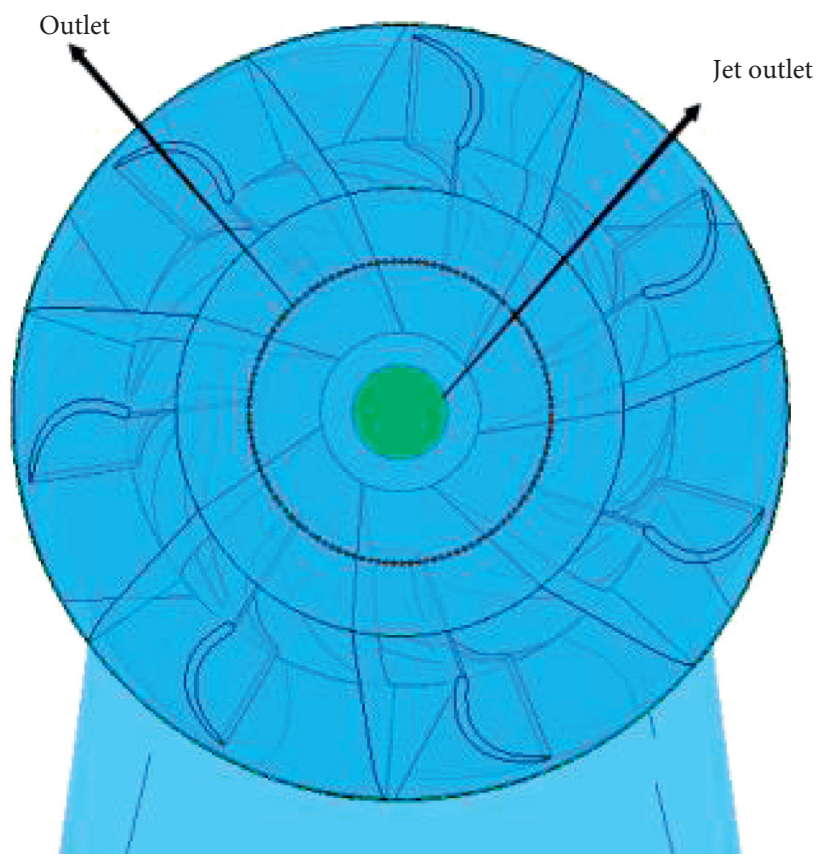

(c)

Figure 2: Computational domain. (a) Overall picture. (b) Partial enlarged view. (c) Partial enlarged view.

coordinate value of each point on the central axis of the nozzle section, and $V z / V o$ represents the ratio of the axial velocity at that point to the axial velocity at that point in the original device, also known as the relative velocity. $V_{j}$ represents the jet velocity.

3.2. Analysis of Internal Flow Field Characteristics. Figure 8 is a graph showing the average velocity and total pressure change of the nozzle under different jet flow rates. It can be clearly seen from the figure that, with the increase of the jet flow rate, the average velocity of the nozzle outlet section increases, and the range of change becomes larger and larger. The change rule of the total pressure of the nozzle section is consistent with the change rule of the average velocity. Figure 9 is a cloud diagram of the axial velocity distribution of the nozzle, Figure 9(a) is a cloud diagram of the axial velocity distribution of the nozzle of the original device, and Figures 9(b)-9(f) are the cloud diagrams of the nozzle axial velocity distribution of the device when the jet velocity is $0 \mathrm{~m} / \mathrm{s}, 4 \mathrm{~m} / \mathrm{s}, 8 \mathrm{~m} / \mathrm{s}, 12 \mathrm{~m} / \mathrm{s}$, and 


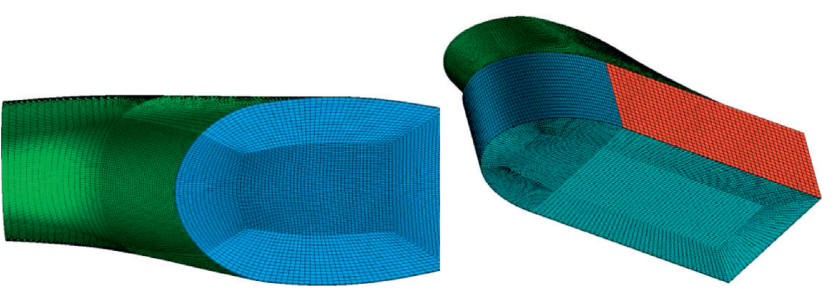

(a)

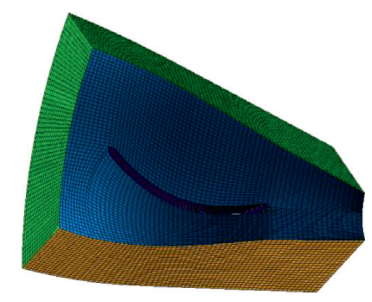

(c)

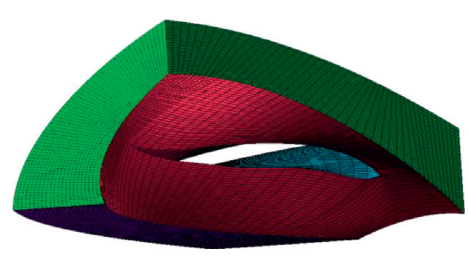

(b)

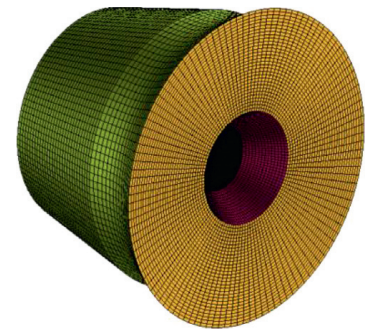

(d)

FIGURE 3: Block structured grid of water jet propulsion pump device. (a) Inlet passage. (b) Impeller. (c) Guide vane. (d) Nozzle.

TABle 1: The number of different impeller domain grids.

\begin{tabular}{lcccc}
\hline Grid & Impeller grid/10,000 & Guide vane and nozzle grid/10,000 & Inlet passage and water grid/10,000 & Total grid/10,000 \\
\hline 1 & 40 & 100 & 160 & 300 \\
2 & 50 & 100 & 160 & 310 \\
3 & 60 & 100 & 160 & 320 \\
4 & 70 & 100 & 160 & 330 \\
5 & 80 & 100 & 160 & 340 \\
6 & 90 & 100 & 160 & 350 \\
\hline
\end{tabular}

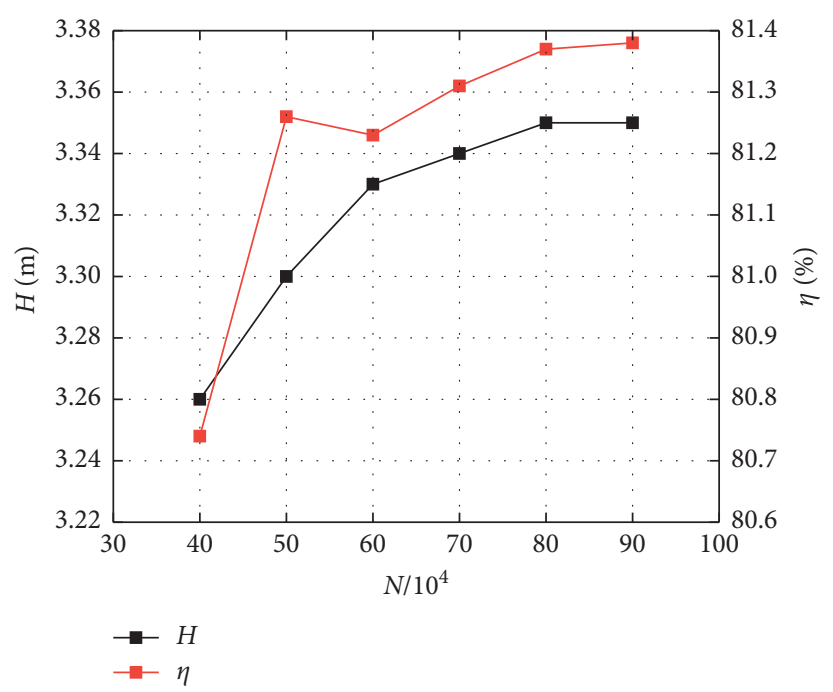

FIGURE 4: Grid independence analysis.

$16 \mathrm{~m} / \mathrm{s}$. Observing the cloud image, it can be found that the central low-velocity area of Figure 9(a) is a bit larger than that of Figure 9(a). The reason is that the original water-conducting cone has changed its shape and cannot play the role of waterconducting, so the water flow velocity will be somewhat loss. The axial velocity distribution uniformity of the nozzle in Figure $9(\mathrm{c})$ is relatively uniform. The reason is that the jet velocity of $4 \mathrm{~m} / \mathrm{s}$ is just close to the water velocity near the water guide cone. When the jet is merged, the axial velocity distribution at the nozzle will be more uniform. The high-speed zone appears in the center of Figure 9(d), Figure 9(e), and Figure 9(f). The reason is that it is affected by the external jet, and as the jet velocity increases, the velocity of the high-speed zone also increases, and the range of the high-speed zone is approximately equal to the jet exit area. In the figure, $v_{\text {jet }}$ represents the size of the jet velocity and its unit is $\mathrm{m} / \mathrm{s}$. 
TABLE 2: Research plan.

\begin{tabular}{|c|c|c|c|c|}
\hline Series & Plan & Speed $(\mathrm{m} / \mathrm{s})$ & Jet velocity $(\mathrm{m} / \mathrm{s})$ & Jet exit area \\
\hline \multirow{5}{*}{ One } & 1 & \multirow{5}{*}{10} & 0 & \multirow{15}{*}{ The jet exit area is $30.77 \%$ of the nozzle exit area } \\
\hline & 2 & & 4 & \\
\hline & 3 & & 8 & \\
\hline & 4 & & 12 & \\
\hline & 5 & & 16 & \\
\hline \multirow{5}{*}{ Two } & 6 & \multirow{5}{*}{8} & 0 & \\
\hline & 7 & & 4 & \\
\hline & 8 & & 8 & \\
\hline & 9 & & 12 & \\
\hline & 10 & & 16 & \\
\hline \multirow{5}{*}{ Three } & 11 & & 0 & \\
\hline & 12 & & 4 & \\
\hline & 13 & 6 & 8 & \\
\hline & 14 & & 12 & \\
\hline & 15 & & 16 & \\
\hline
\end{tabular}

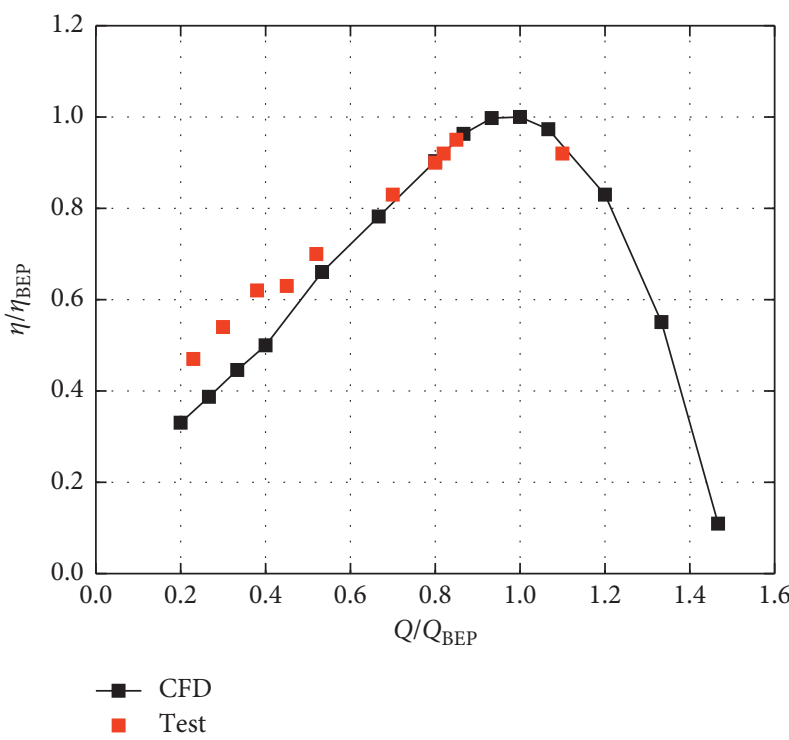

(a)

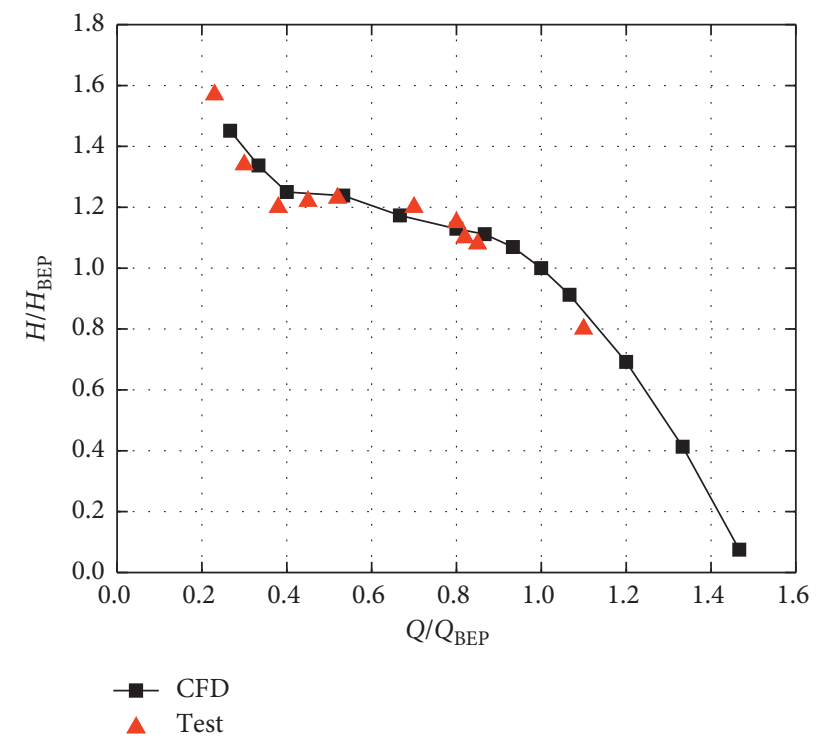

(b)

FIgURE 5: Comparison of numerical simulation and experimental results. (a) Q- $\eta$ curve. (b) Q-H curve.

Figure 10 is the velocity distribution cloud diagram of the longitudinal section of the pump section, Figure 10(a) is the velocity distribution cloud diagram of the longitudinal section of the original pump section, and Figures $10(\mathrm{~b})$ $10(f)$ are the vertical section velocity distribution cloud diagrams of the pump section of the device when the jet velocity is $0 \mathrm{~m} / \mathrm{s}, 4 \mathrm{~m} / \mathrm{s}, 8 \mathrm{~m} / \mathrm{s}, 12 \mathrm{~m} / \mathrm{s}$, and $16 \mathrm{~m} / \mathrm{s}$. Observing the cloud picture, we can find that with the increase of jet flow, the velocity from the guide vane body to the exit section becomes larger and larger, indicating that the water velocity in this section is significantly affected by the external jet.

Figure 11 is a streamline diagram of the jet exit at different jet velocities, Figure 11(a) is the streamlines before the jet exit when the jet velocity is $0 \mathrm{~m} / \mathrm{s}, 4 \mathrm{~m} / \mathrm{s}, 8 \mathrm{~m} / \mathrm{s}, 12 \mathrm{~m} / \mathrm{s}$, and $16 \mathrm{~m} / \mathrm{s}$. Observing Figures 11(a)-11(e), it can be seen that when the jet velocity is $0 \mathrm{~m} / \mathrm{s}$, the flow state of the water in front of the jet outlet is turbulent and spiral. As the jet velocity increases, the spiral phenomenon gradually disappears. The jet velocity is $16 \mathrm{~m} / \mathrm{s}$, and the external jet is basically horizontal and straight. Moreover, it can be seen from Figures 11(a) to 11(e) that the water velocity in front of the jet exit section gradually increases, but the increase is not large and relatively uniform. The reason is that the velocity of the two streams increases after the convergence of the two streams.

3.3. Eddy Dynamic Analysis. In order to study the motion of the vortices in the water jet propulsion pump device under different jet flow rates, the turbulent kinetic energy equation and the transport equation of the turbulent dissipation rate are used to conduct in-depth research and analysis on the internal vortex of the water jet propulsion pump device with the jet flow. In the standard $k-\varepsilon$ model, the transport equations of turbulent kinetic energy $k$ and turbulent energy dissipation $\varepsilon$ are as follows: 


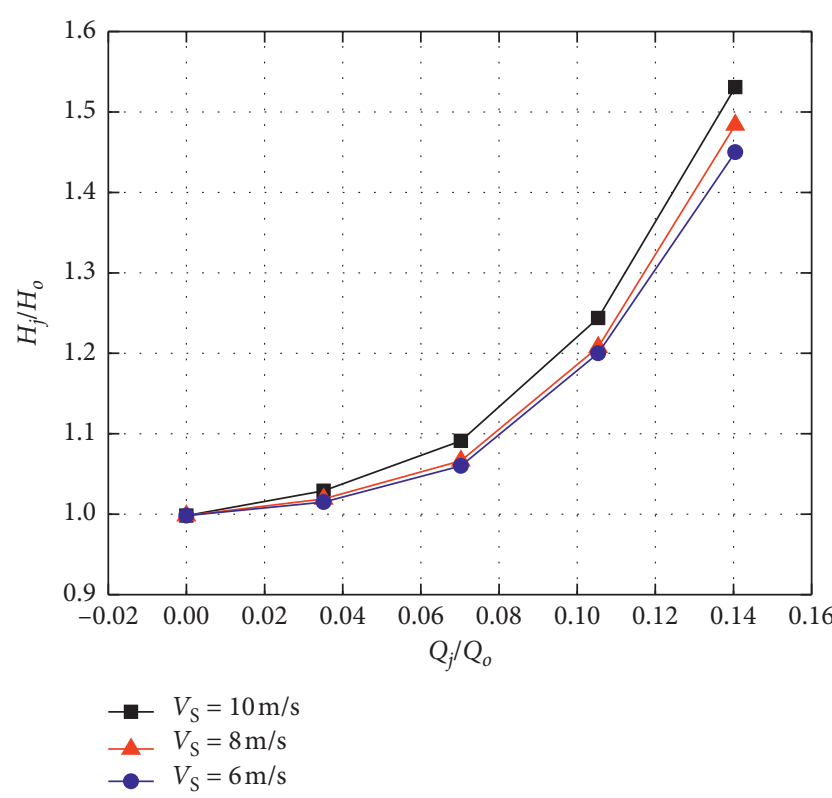

Figure 6: Relation diagram of head and jet flow.

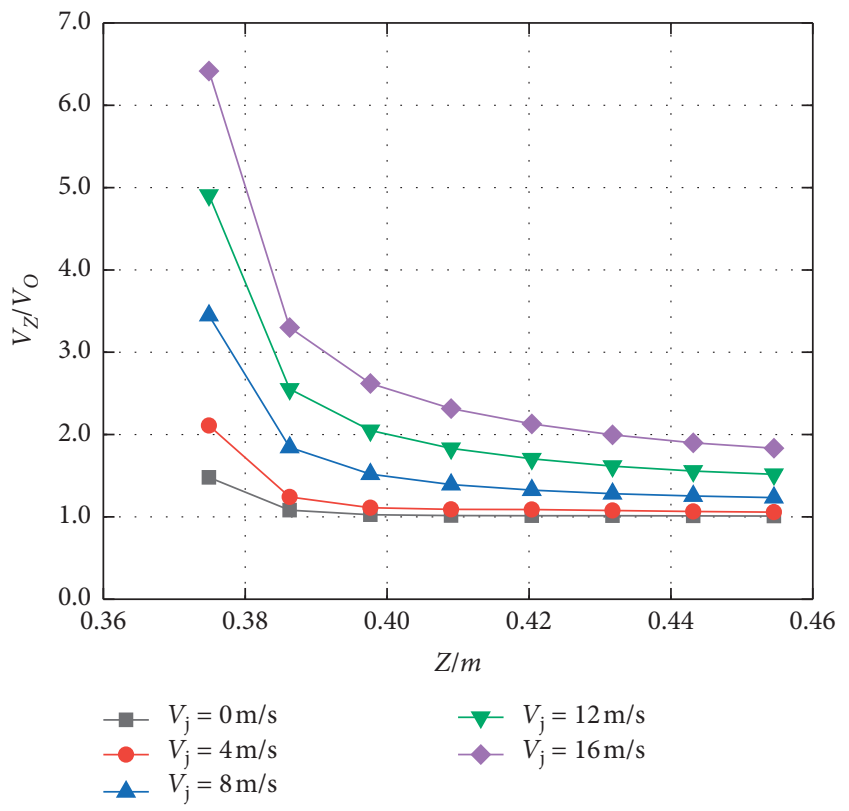

FIgURE 7: Speed change on the central axis.

$$
\begin{aligned}
& \frac{\partial(\rho k)}{\partial t}+\frac{\partial\left(\rho k u_{j}\right)}{\partial x_{j}}=\frac{\partial}{\partial x_{j}}\left[\left(\mu+\frac{\mu_{t}}{\sigma_{k}}\right) \frac{\partial k}{\partial x_{j}}\right]+\rho\left(Q_{k}-\varepsilon\right), \\
& \frac{\partial(\rho \varepsilon)}{\partial t_{j}}+\frac{\partial\left(\rho \varepsilon u_{j}\right)}{\partial x_{j}}=\frac{\partial}{\partial x_{j}}\left[\left(\mu+\frac{\mu_{t}}{\sigma_{\varepsilon}}\right) \frac{\partial \varepsilon}{\partial x_{j}}\right]+\rho \frac{\varepsilon}{k}\left(C_{1} P_{k}-C_{2} \varepsilon\right),
\end{aligned}
$$

where $Q_{k}$ is the generation term of turbulent kinetic energy; $\mu_{t}$ is the viscosity of turbulent flow; $k$ is turbulent kinetic energy, which refers to the amount of change in velocity fluctuations, in $\mathrm{m}^{2} / \mathrm{s}^{2} ; \varepsilon$ is turbulent energy dissipation, which refers to the rate of dissipation of velocity

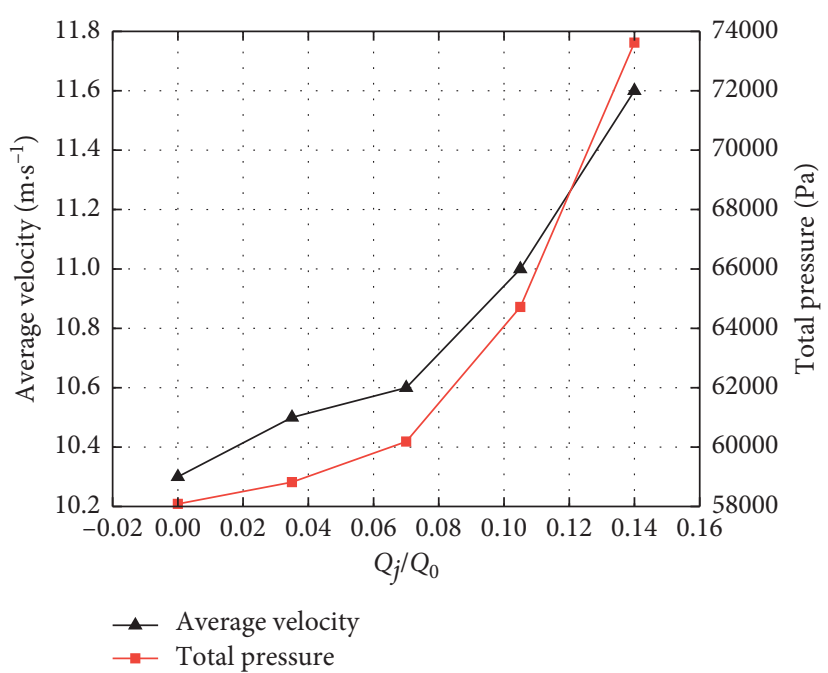

FIGURE 8: Variations of average nozzle velocity and total pressure under different jet flow rates.

fluctuations, and its unit is $\mathrm{m}^{2} / \mathrm{s}^{3}$; and constant $C_{1}=1.44$, $C_{2}=1.92, \sigma_{k}=1.0, \sigma_{\varepsilon}=1.0$.

3.3.1. Vorticity Analysis. Vorticity is one of the most important physical quantities describing the motion of a vortex. It is defined as the curl of the fluid velocity vector. The unit of vorticity is one second $\left(\mathrm{s}^{-1}\right)$. Vortex usually uses vorticity to measure its strength and direction. In the fluid, as long as there is a "vortex source," vortices of different sizes will be produced. Therefore, the positive or negative of the vorticity value represents different vortex rotation directions, and the absolute value of the vorticity represents the probability of vortex occurrence.

Figure 12 shows the average vorticity and maximum vorticity changes of the nozzle under different jet flow rates. Among them, as the jet flow increases, the absolute value of the average vortex of the nozzle gradually decreases, indicating that the probability of vortex occurrence on the nozzle section gradually decreases. This is because the high-speed jet affects the original moving water flow, which reduces it to a certain extent. The generation of vortex is in the nozzle section. The absolute value of the maximum vorticity shows a gradually increasing trend overall. It can be seen from the figure that the maximum vorticity changes little when the relative flow rate of the jet is between 0.04 and 0.10.

The vorticity distribution cloud diagram of the nozzle outlet section under different jet flow rates is shown in Figure 13. When the additional jet flow rate is 0 , a large positive vortex appears in the center area of the nozzle outlet section, and the blue area shown in the figure appears around it The direction of the vortex is opposite to the central area. With the increase of jet flow, the vortex in the central area of the nozzle exit section gradually decreases, indicating that the vortex phenomenon is not obvious when the jet flow is large.

The vortex distribution cloud diagram of the longitudinal section of the nozzle section under different jet flow rates is shown in Figure 14. It can be found that, as the jet 


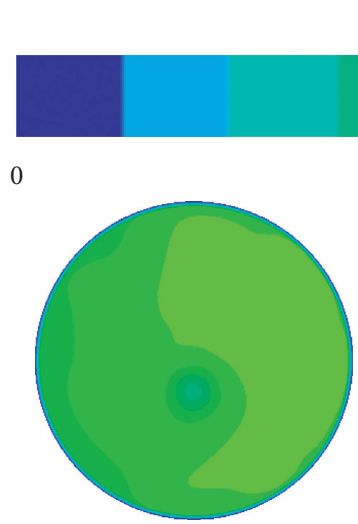

(a)

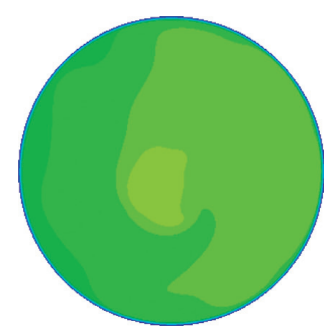

(d)

Velocity w $\left(\mathrm{m} \mathrm{s}^{-1}\right)$

10

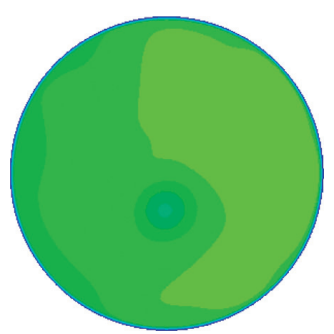

(b)

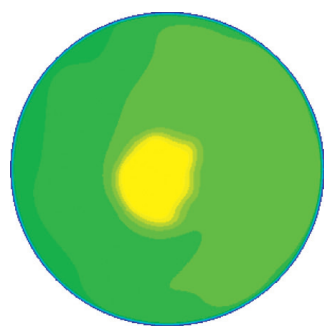

(e)
20

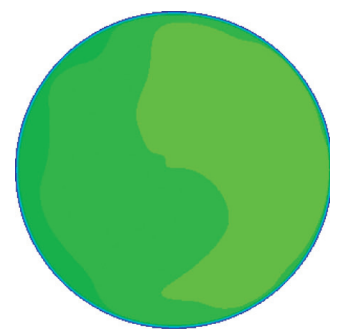

(c)

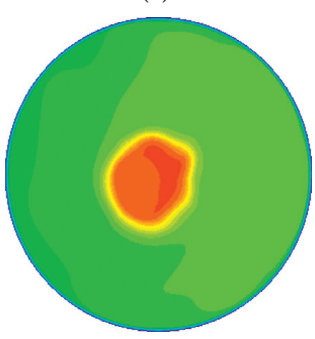

(f)

Figure 9: Axial velocity distribution cloud diagram of the nozzle. (a) Original device, (b) $v_{\text {jet }}=0$, (c) $v_{\text {jet }}=4$, (d) $v_{\text {jet }}=8$, (e) $v_{\text {jet }}=12$, and (f) $v_{\text {jet }}=16\left(\mathrm{~m} \mathrm{~s}^{-1}\right)$.

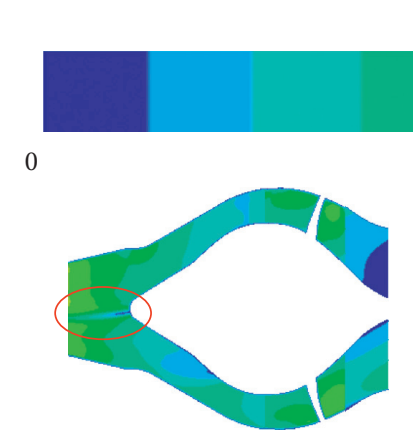

(a)

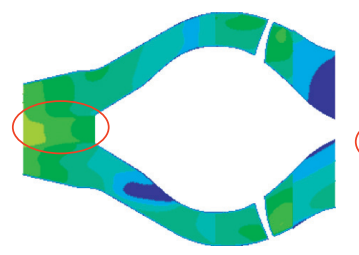

(d)

\section{Velocity $\left(\mathrm{m} \mathrm{s}^{-1}\right)$}

9.5

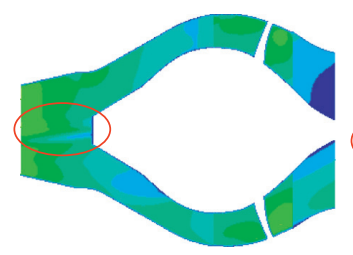

(b)

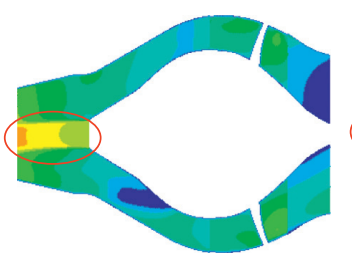

(e)

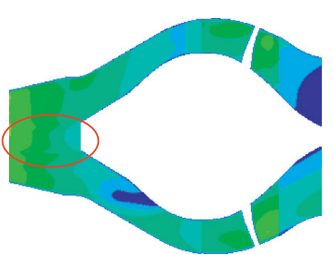

(c)

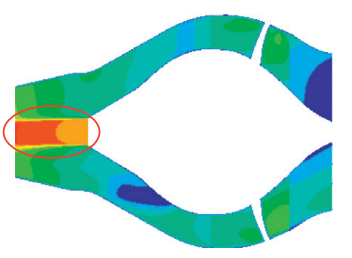

(f)

FIGURE 10: The velocity distribution cloud diagram of the longitudinal section of the pump section. (a) Original device, (b) $v_{\text {jet }}=0$, (c) $v_{\text {jet }}=4$, (d) $v_{\text {jet }}=8$, (e) $v_{\text {jet }}=12$, (f) $v_{\text {jet }}=16\left(\mathrm{~m} \mathrm{~s}^{-1}\right)$.

flow rate increases, the vortex generated by the water flow in front of the water guide cone gradually decreases and changes direction. Due to the influence of the external jet, some large eddies appear around the jet exit path, and these eddies gradually decrease with the increase of the jet flow.
3.3.2. Turbulent Kinetic Energy Analysis. Turbulent kinetic energy is $1 / 2$ of the product of turbulent velocity fluctuation variance and fluid mass. The change of total kinetic energy of turbulence over time reflects the net income and expenditure of turbulence energy and is an indicator to measure the 

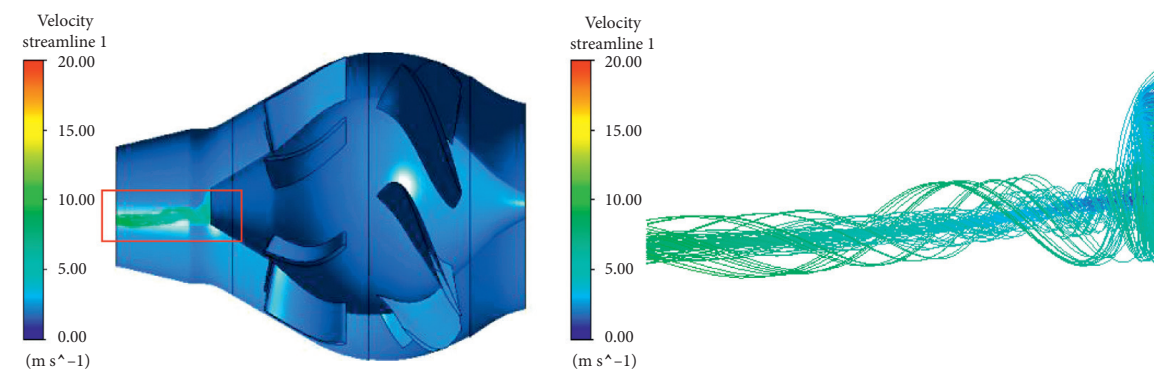

(a)
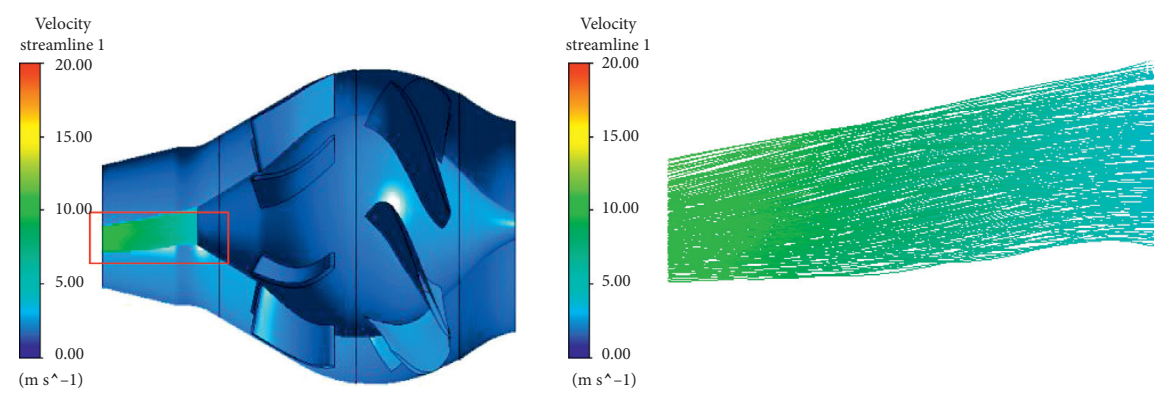

(b)
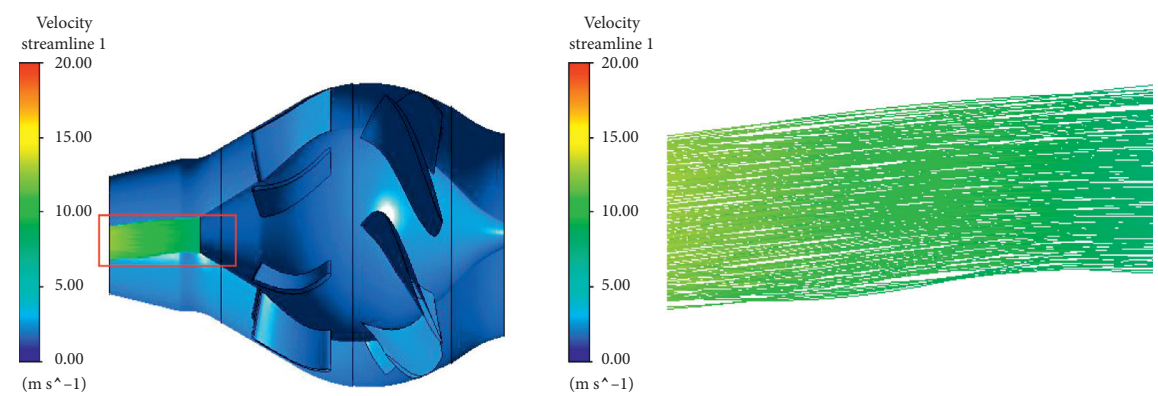

(c)
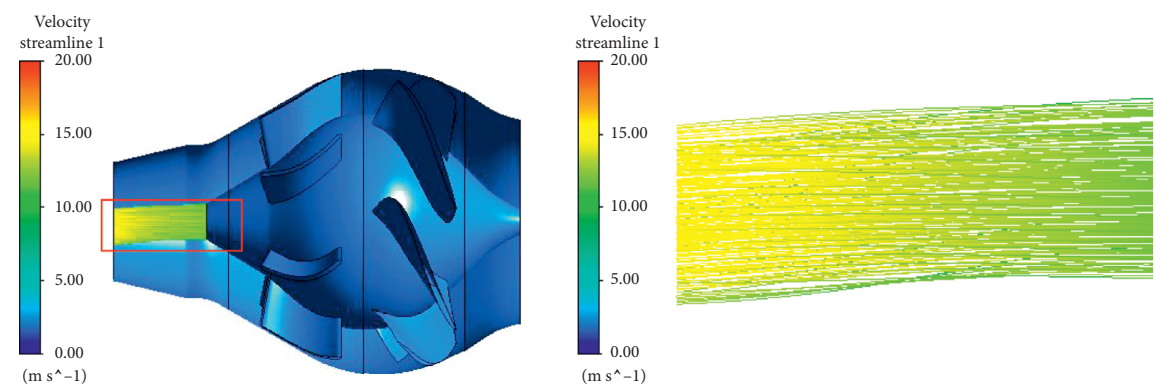

(d)
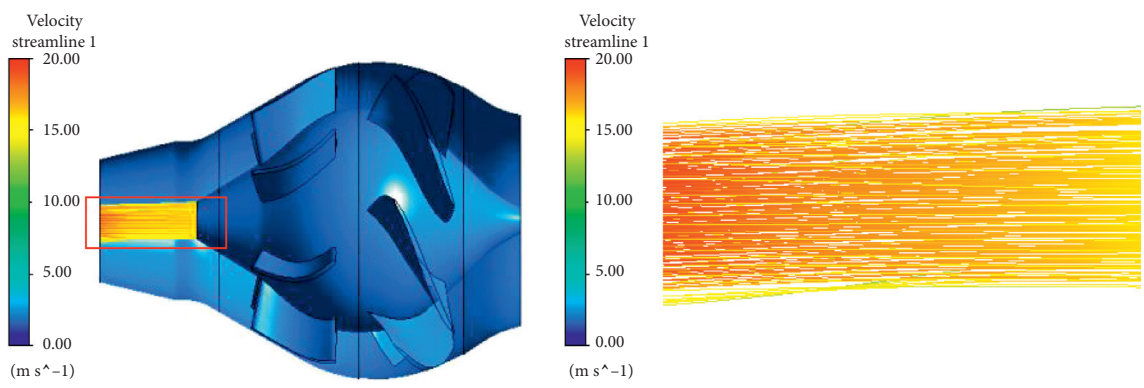

(e)

FIGURE 11: Streamline diagram in front of the outlet of the jet tube at different jet velocities: (a) $v_{\text {jet }}=0$, (b) $v_{\text {jet }}=4$, (c) $v_{\text {jet }}=8$, (d) $v_{\text {jet }}=12$, (e) $v_{\text {jet }}=16\left(\mathrm{~m} \mathrm{~s}^{-1}\right)$. 


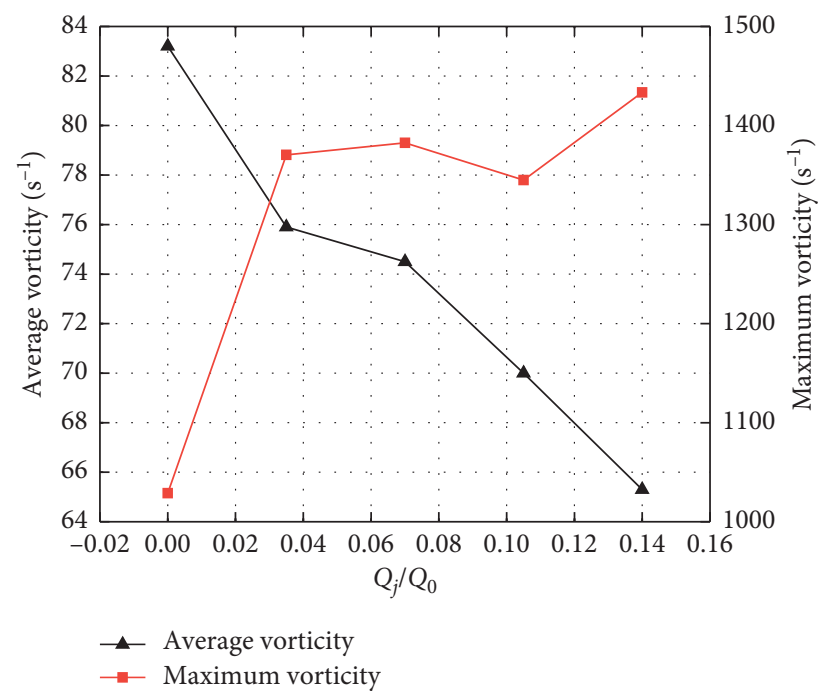

FIgURE 12: The average vorticity and maximum vorticity changes of nozzles under different jet flow rates.

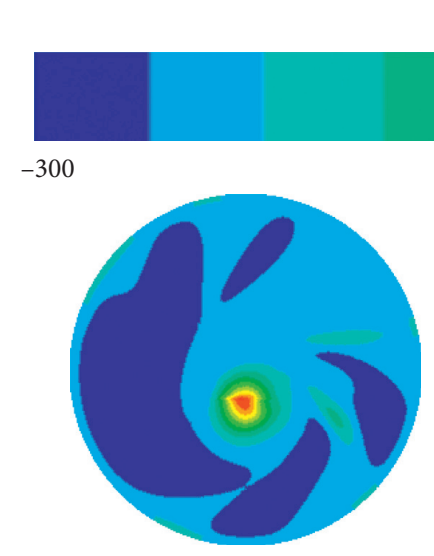

(a)

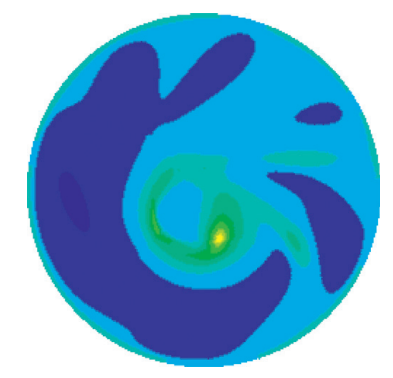

(d)

Vorticity w $\left[\mathrm{s}^{-1}\right]$

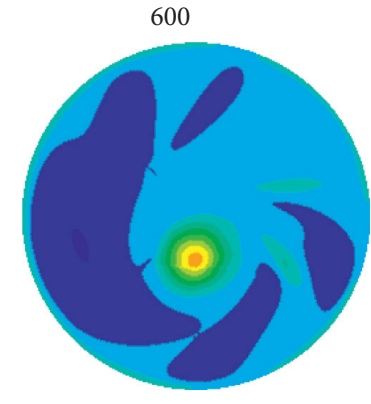

(b)

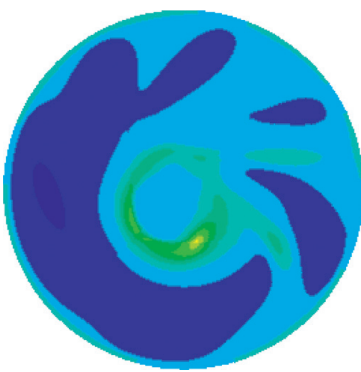

(e)

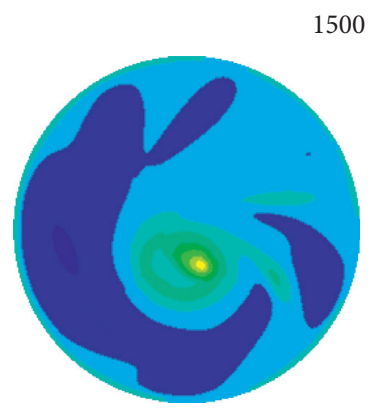

(c)

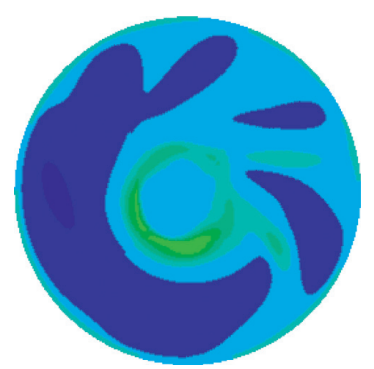

(f)

FiguRE 13: Cloud diagram of vorticity distribution in the section of the outlet of the nozzle. (a) Original device, (b) $v_{\text {jet }}=0$, (c) $v_{\text {jet }}=4$, (d) $v_{\text {jet }}=8$, (e) $v_{\text {jet }}=12$, (f) $v_{\text {jet }}=16$.

development or decline of turbulence. The variance of turbulent diffusion is proportional to the component turbulence energy, and it is an important indicator to measure the turbulent mixing ability. Turbulent flow energy is represented by $k$. Turbulent energy dissipation rate refers to the rate at which turbulent energy is converted into kinetic energy of molecular thermal motion under the action of molecular viscosity. It is usually measured by the turbulent flow energy lost per unit mass of fluid in a unit time, expressed by $\varepsilon$. The turbulent velocity has random fluctuations in space, thus forming a significant velocity gradient. Under the action of the molecular viscous force, the turbulent energy is continuously converted into the kinetic energy of molecular motion through internal friction: 


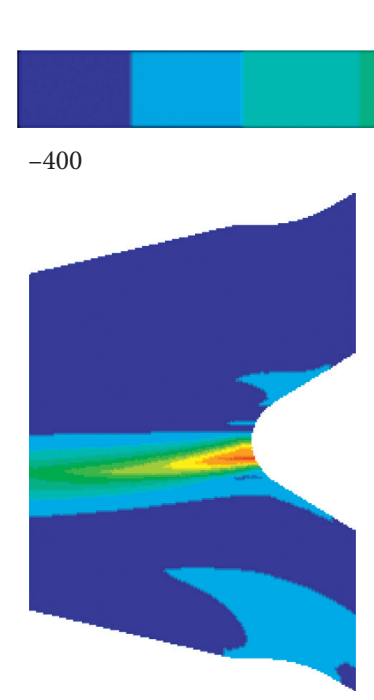

(a)

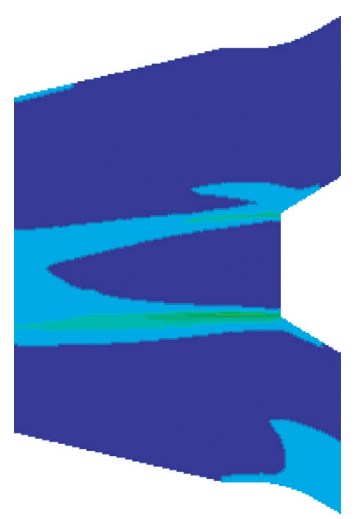

(d)

\section{Vorticity w $\left(\mathrm{s}^{-1}\right)$}

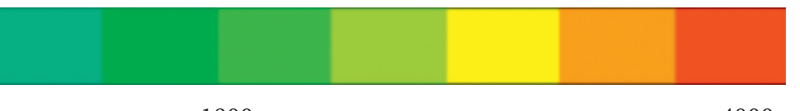

1800

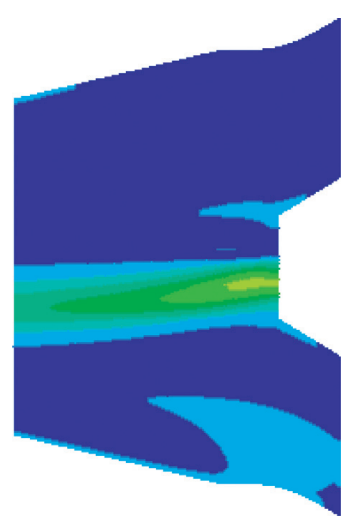

(b)

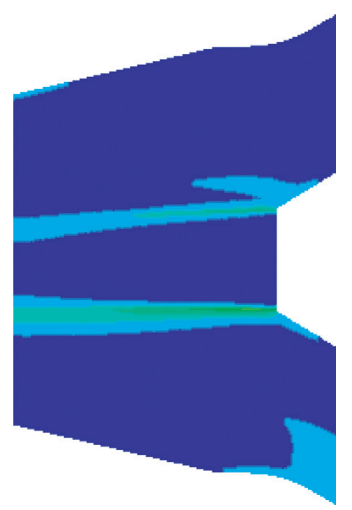

(e)

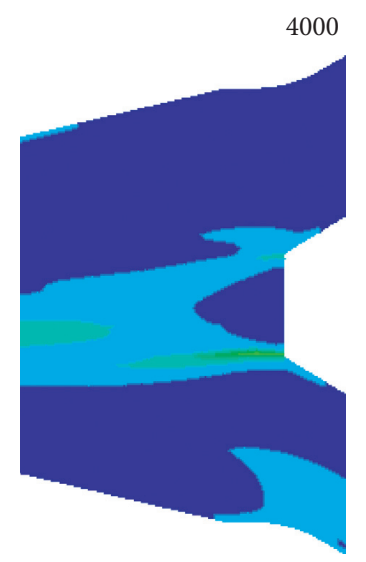

(c)

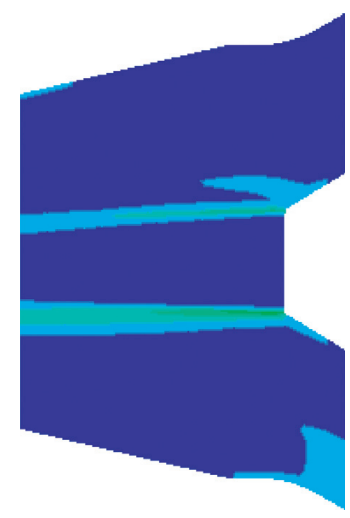

(f)

Figure 14: Vortex distribution cloud diagram of the longitudinal section of the nozzle section. (a) Original device, (b) $v_{\text {jet }}=0$, (c) $v_{\text {jet }}=4$, (d) $v_{\text {jet }}=8$, (e) $v_{\text {jet }}=12$, (f) $v_{\text {jet }}=16\left(\mathrm{~m} \mathrm{~s}^{-1}\right)$.

$$
\begin{aligned}
& k=1.5 *(u * l)^{2}, \\
& E=\frac{C u^{0.75} * k^{1.5}}{l} .
\end{aligned}
$$

In the formula, $u$ is the average velocity, $I$ is the turbulence intensity, and $\mathrm{Cu}$ is usually 0.09 .

Figure 15 is a graph showing the change of turbulent kinetic energy and turbulent energy dissipation of the nozzle under different jet flow rates. It can be seen from the figure that, as the jet flow increases, the turbulent kinetic energy does not change much, indicating that the flow state is relatively stable. The turbulent energy dissipation value of the nozzle section first decreases and then increases, indicating that the turbulent energy dissipation is fast under the high-speed jet.

The distribution of turbulent kinetic energy at the outlet section of the nozzle in Figure 16 shows the following: (1) when no jet is added, the distribution of turbulent kinetic energy is consistent with the distribution trend of the original device. Larger turbulent kinetic energy appears in the center of the nozzle section and moves to the surroundings. It is reduced in the shape of a volute, which is mainly due to the collision of water flow at the water guide cone during the movement of the fluid, and there is a large energy loss. (2) When the external jet is operating, there is almost no turbulent kinetic energy generated in the center of the nozzle section. As the flow of the external jet increases, obvious turbulent kinetic energy begins to appear around the center, which is mainly when the external jet merges with the original water flow, caused by the loss of water flow.

The turbulent kinetic energy distribution cloud diagram of the longitudinal section of the nozzle section under different jet flow rates is shown in Figure 17. With the increase of the jet flow rate, the water loss at the front of the jet exit section decreases, the turbulent kinetic energy decreases, and the turbulent kinetic energy under the jet is larger than above the jet. This is because the gravity of the jet water flow causes the collision and impact between the jet and the original water flow to be more severe, and the hydraulic loss is greater.

3.4. Analysis of Mechanical Characteristics. Figure 18 is a graph showing the change in the growth rate of the nozzle axial force under different jet flow rates. It can be clearly seen 


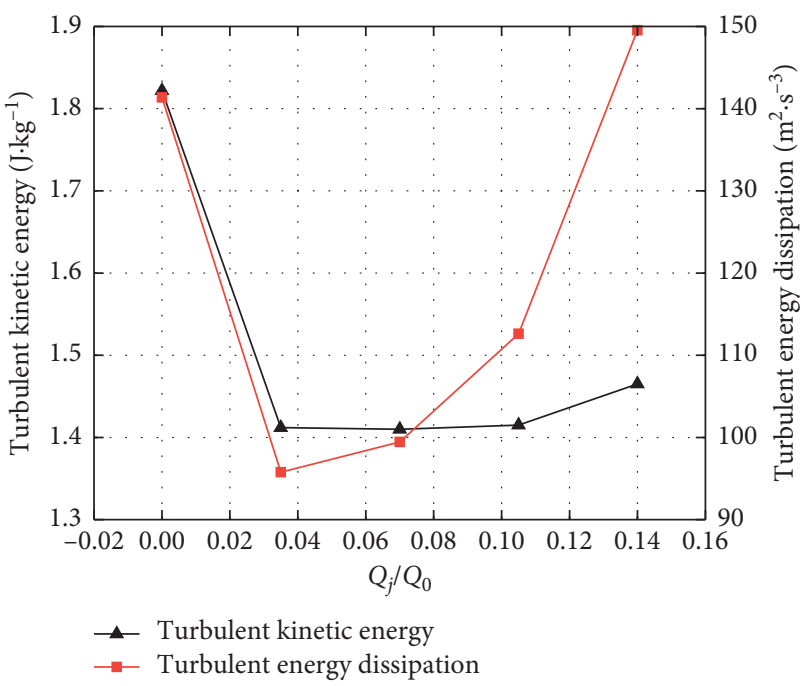

Figure 15: The change of turbulent kinetic energy and turbulent energy dissipation of the nozzle under different jet flow rates.

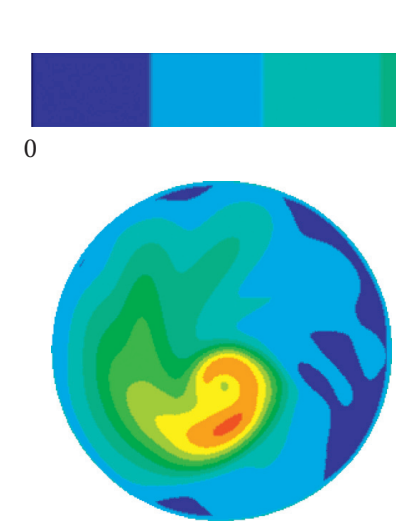

(a)

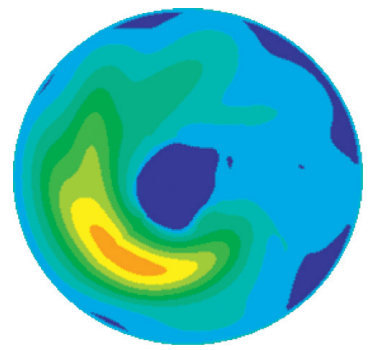

(d)

Turbulence kinetic energy $\left(\mathrm{m}^{2} \mathrm{~s}^{2}\right)$

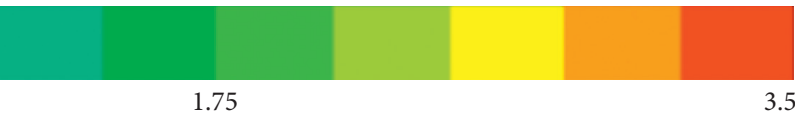

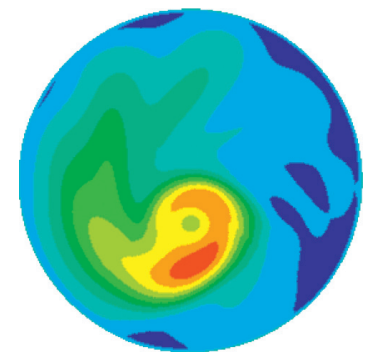

(b)

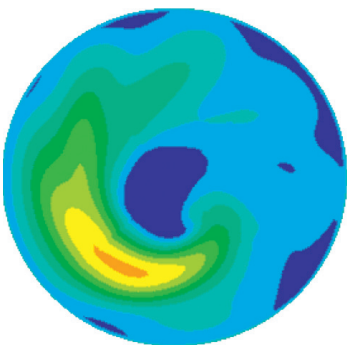

(e)

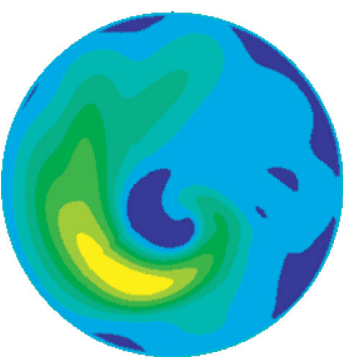

(c)

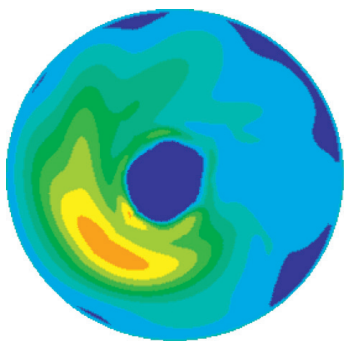

(f)

Figure 16: Cloud diagram of turbulent kinetic energy distribution at the outlet section of the nozzle. (a) Original device, $(\mathrm{b}) v_{\mathrm{jet}}=0$, (c) $v_{\text {jet }}=4$, (d) $v_{\text {jet }}=8$, (e) $v_{\text {jet }}=12$, (f) $v_{\text {jet }}=16\left(\mathrm{~m} \mathrm{~s}^{-1}\right)$.

from the figure that when the jet flow rate is small, the axial force change rate is not large, but with the increase of the jet flow rate, the axial force growth rate increases sharply, and the axial force changes more and more. Among the schemes studied, the maximum rate of change of the nozzle axial force reached $9.2 \%$. Since the axial force is a calculation variable that comes with CFX postprocessing, it can be easily obtained.
The thrust of the device is not easy to calculate, but it has a positive correlation with the axial force, so the trend of the thrust of the device can be inferred. It can be found that the greater the flow rate of the external jet, the greater the increase in the thrust of the device, which just verifies the fact that the additional jet device does increase the thrust compared to the original device and increases the ship's sailing speed. 


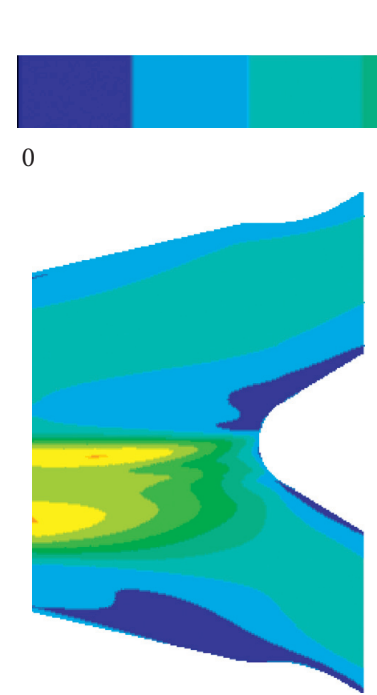

(a)

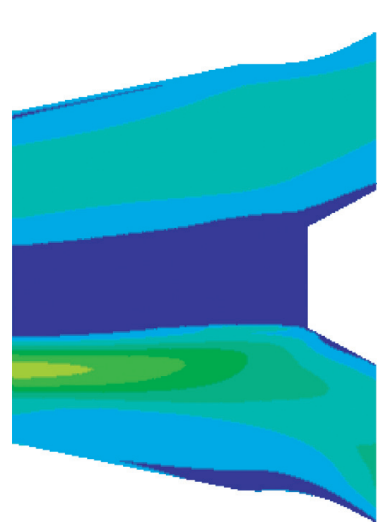

(d)

Turbulence kinetic energy $\left(\mathrm{m}^{2} \mathrm{~s}^{2}\right)$

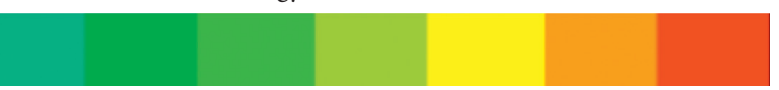

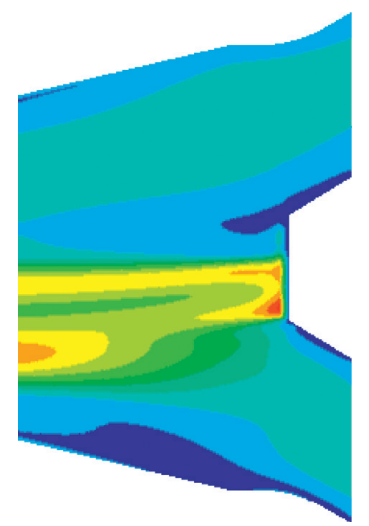

(b)

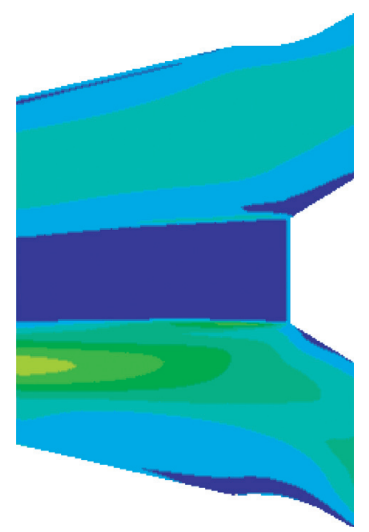

(e)

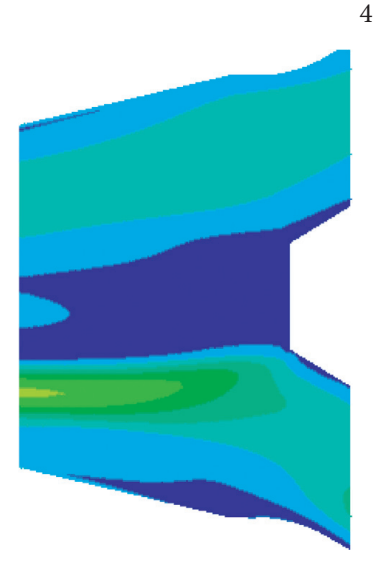

(c)

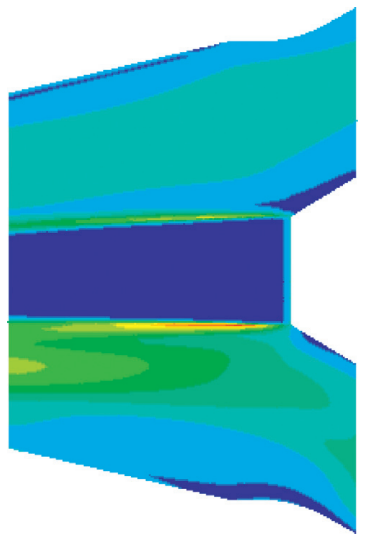

(f)

FIGURE 17: Cloud diagram of turbulent kinetic energy distribution in the longitudinal section of the nozzle section. (a) Original device, (b) $v_{\text {jet }}=0$, (c) $v_{\text {jet }}=4$, (d) $v_{\text {jet }}=8$, (e) $v_{\text {jet }}=12$, (f) $v_{\text {jet }}=16\left(\mathrm{~m} \mathrm{~s}^{-1}\right)$.

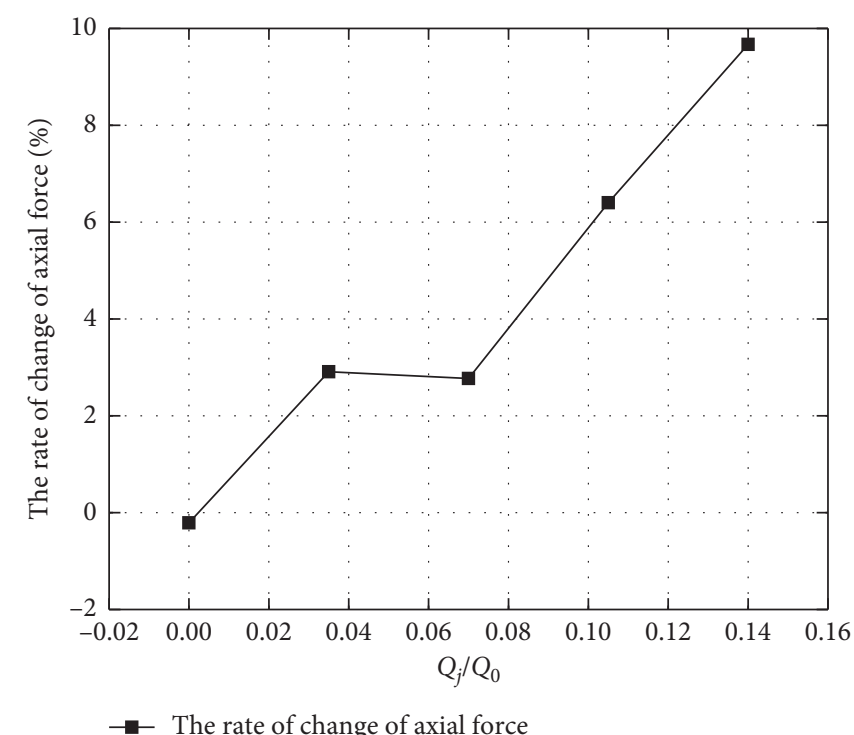

FIgURE 18: The growth rate of the nozzle axial force under different jet flow rates. 


\section{Conclusion}

Based on the standard $k-\varepsilon$ turbulence model, the hydraulic performance, flow field characteristics, vorticity characteristics, and mechanical properties of the water jet propulsion pump device with external jets under different jet flow rates and speeds are numerically calculated and analyzed. The conclusions are as follows:

(1) The numerically calculated efficiency-flow curve and head-flow curve are in good agreement with the experimental test results in the literature.

(2) With the increase of jet flow rate, the average velocity of the nozzle exit section increases, and the range of change becomes larger and larger. The change rule of the total pressure of the nozzle section is consistent with the change rule of the average velocity.

(3) When the flow rate of the external jet is small, the flow state of the water in front of the jet tube outlet is turbulent and spiral. With the increase of the flow of the external jet, the spiral phenomenon gradually disappears. When the flow of the external jet is greater than a certain value, the external jet is added. The project is basically horizontal and straight.

(4) As the jet flow increases, the absolute value of the average vortex of the nozzle gradually decreases, indicating that the probability of vortex occurrence at the nozzle section gradually decreases. This is because the high-speed jet affects the original moving water flow, which reduces the generation of vortices at the nozzle section to a certain extent. The absolute value of the maximum vorticity shows a gradually increasing trend overall. When the relative flow rate of the jet is between 0.04 and 0.10 , the maximum vorticity does not change much.

(5) When no jet is added, the distribution of turbulent kinetic energy is consistent with that of the original device. Larger turbulent kinetic energy appears in the center of the nozzle section and decreases in the shape of a volute around it. This is mainly due to the guiding of the fluid during the movement. Water flow collision occurs at the water cone, and there is a large energy loss. With the increase of jet flow rate, the turbulent kinetic energy does not change much, indicating that the flow pattern is relatively stable. The turbulent energy dissipation value of the nozzle section first decreases and then increases, indicating that the turbulent energy dissipation is fast under the high-speed jet.

(6) When the jet flow rate is small, the axial force change rate is not large, but with the increase of the jet flow rate, the axial force growth rate increases sharply, and the axial force changes more and more. In the scheme studied in this paper, the nozzle axis maximum change rate of the direction force reached $9.2 \%$. And the greater the flow rate of the external jet, the greater the increase in thrust of the device.
In summary, when a high-speed water jet is injected into a moving water body, it will cause turbulence in the moving water body. With the increase of the jet flow rate, the turbulence phenomenon will be improved. The axial force and thrust of the device will obviously increase when the two water streams merge and spray, and they will increase with the increase of the jet flow rate. Due to my limited level, the research in the field of this additional jet is not very thorough, but I hope to attract more scholars' attention, so as to further study the interaction mechanism between this highspeed jet and the moving water body. Especially for the research on the thrust performance of the entire device, there is still a need for further breakthroughs.

\section{Data Availability}

The curve data used to support the findings of this study are available from the corresponding author upon request.

\section{Conflicts of Interest}

The authors declare that there are no conflicts of interest regarding the publication of this paper.

\section{References}

[1] C. Liu, Y. Wang, and J. Ding, "Overview of water jet propulsion research," Ship Engineering, vol. 8, no. 4, pp. 49-52, 2006.

[2] P. Jin, Ship Water Jet Propulsion, National Defense Industry Press, Beijing, China, 1986.

[3] C. Liu, Y. Wang, J. Ding et al., "Overview of water jet propulsion research," Ship Engineering, vol. 6, no. 4, pp. 49-52, 2006.

[4] L. Zhou, W. Wang, J. Hang, W. Shi, H. Yan, and Y. Zhu, "Numerical investigation of a high-speed electrical submersible pump with different end clearances," Water, vol. 12, no. 4, p. 1116, 2020.

[5] L. Shi, J. Zhu, F. Tang, and C. Wang, "Multi-disciplinary optimization design of axial-flow pump impellers based on the approximation model," Energies, vol. 13, no. 4, p. 779, 2020.

[6] L. Zhou, K. Deshpande, X. Zhang, and R. K. Agarwal, "Process simulation of chemical looping combustion using ASPEN plus for a mixture of biomass and coal with various oxygen carriers," Energy, vol. 195, Article ID 116955, 2020.

[7] L. Zhou, C. Han, L. Bai et al., "Agarwal. numerical and experimental study of multiphase transient core-annular flow patterns in a spouted bed," ASME Journal of Energy Resource Technology, vol. 142, no. 9, Article ID 092104, 2020.

[8] J. Zhang, Z. Xia, L. Huang et al., "Summary of research on underwater gas-liquid two-phase propulsion technology," Ship Mechanics, vol. 24, no. 11, pp. 1507-1516, 2020.

[9] H. Meng, Y. Wang, Y. Yu et al., "Research progress of mixing time in jet mixing equipment," Chemical Industry Progress, vol. 31, no. 12, pp. 2615-2625, 2012.

[10] Z. Yu, T. Zhan, J. Li et al., "Research on the influence of pressure chamber of waterjet propeller based on high-speed jet on thrust," China Water Transport (Second Half), vol. 14, no. 3, pp. 142-144, 2014.

[11] H. Wu, D. Tan, R. L. Miorini, and J. Katz, “Three-dimensional flow structures and associated turbulence in the tip region of a 
waterjet pump rotor blade," Experiments in Fluids, vol. 51, no. 6, pp. 1721-1737, 2011.

[12] W. Jiao, L. Cheng, D. Zhang et al., "Investigation of key parameters for the hydraulic optimization of the inlet duct based on a whole waterjet propulsion pump system," Transactions of Famena, vol. 45, no. 1, pp. 1-18, 2021.

[13] S. A. Kinnas and H. Lee, "Prediction of cavitating waterjet propulsor perfomance using a boundary element method," in Proceedings of the 9th Intemnational Conference on Numerical Ship Hydrodynamics, Ann Arbor, MI, USA, October 2007.

[14] C. Wang, W. Shi, X. Wang et al., "Optimal design of multistage centrifugal pump based on the combined energy loss model and computational fluid dynamics," Applied Energy, vol. 187, pp. 10-26, 2017.

[15] C. Wang, X. Chen, N. Qiu et al., "Numerical and experimental study on the pressure fluctuation, vibration, and noise of multi-stage pump with radial diffuser," Journal of the Brazilian Society of Mechanical Sciences and Engineering, vol. 40, p. 481, 2018.

[16] X. He, Y. Zhang, C. Wang et al., "Influence of critical wall roughness on the performance of double-channel sewage pump," Energies, vol. 13, no. 2, p. 464, 2020.

[17] D. Zhang, W. Jiao, L. Cheng et al., "Experimental study on the evolution process of the roof-attached vortex of the closed sump," Renewable Energy, vol. 164, pp. 1029-1038, 2021.

[18] H. L. Wang, B. Long, Y. Yang, Y. Xiao, and C. Wang, "Modelling the influence of inlet angle change on the performance of submersible well pumps," International Journal of Simulation Modelling, vol. 19, no. 1, pp. 100-111, 2020.

[19] H. Wang, B. Long, C. Wang, C. Han, and L. Li, "Effects of the impeller blade with a slot structure on the centrifugal pump performance," Energies, vol. 13, no. 7, p. 1628, 2020.

[20] H. L. Wang, Q. X. Hu, Y. Yang, and C. Wang, "Performance differences of electrical submersible pump under variable speed schemes," International Journal of Simulation Modelling, vol. 20, no. 1, pp. 76-86, 2021.

[21] J. Zhou, M. Zhao, C. Wang et al., "Influence of different lateral bending angles on the flow pattern of pumping station lateral inflow," Shock and Vibration, vol. 2021, Article ID 6653001, 9 pages, 2021.

[22] J. Zhou, M. Zhao, C. Wang et al., "Optimal design of diversion piers of lateral intake pumping station based on orthogonal test," Shock and Vibration, vol. 2021, Article ID 6616456, 9 pages, 2021.

[23] X. Duan, Y. Wang, Y. Su et al., "Joint classification and recognition of cavitation characteristics of water jet propulsion pump," Journal of Shanghai Jiaotong University, vol. 44, no. 9, pp. 1322-1426, 2011.

[24] S. Jin, "Three-dimensional design and numerical experiment of Francis water jet propulsion pump," Journal of Harbin Engineering University, vol. 33, no. 10, pp. 1223-1227, 2012.

[25] H. Wang, Z. Qian, D. Zhang, T. Wang, and C. Wang, "Numerical study of the normal impinging water jet at different impinging height, based on wray-agarwal turbulence model," Energies, vol. 13, no. 7, p. 1744, 2020.

[26] S. Chang and Y. Wang, "Performance prediction of waterjet propulsion using k-e turbulence model," Journal of Huazhong University of Science and Technology (Natural Science Edition), vol. 40 , no. 4 , p. 89, 2012.

[27] C. Xia, L. Cheng, C. Luo et al., "Hydraulic characteristics and measurement of rotating stall suppression in a waterjet propulsion system," Transactions of FAMENA, vol. 42, no. 4, pp. 85-100, 2018.
[28] L. Shi, Research on Cfd Grid Error Based on Richardson Extrapolation Method, North China Electric Power University, Beijing, China, 2011. 